\title{
The numerical analysis of the unsteady flow in the three - dimensional multiphase mixing flow field
}

\author{
Chao Hui ${ }^{1, a}$, Juan $\mathrm{Xu}^{1, \mathrm{~b}}$ and Haiyan Bie ${ }^{2}$ \\ ${ }^{1}$ Shandong Provincial Key Laboratory of Ocean Environment Monitoring Technology, Institute of \\ Oceanographic Instrumentation, Shandong Academy of Sciences, Zhejiang Road 28, Qingdao \\ 266001, China \\ ${ }^{2}$ College of Chemistry and Chemical Engineering, Ocean University of China

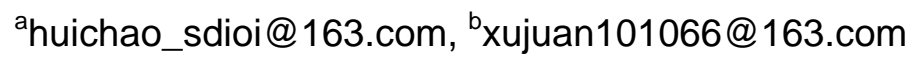

Keywords: multiphase mixing; large eddy simulation; mixture model; flow characteristic; numerical analysis

\begin{abstract}
Unsteady flow and the interface distribution in the stirred tank are studied by the large eddy simulation and multiphase flow model. The result shows that the two-phase interface starts from the paddle. Finally inverted cone interface is generated by the screw move. With mixing time passing by, the interface rotates with the paddle. Centrifugal effect is more obvious along with the speed rise. There is an apparently circulation area among the blades. Velocity near the blade tip is high. And a large number of small vortexes are formed because of the strong turbulence. Then fuels around are carried to other areas by the vortex motion. There are contrary velocity peeks between the hub and the blade tip. And with the speed increasing, difference between the radial velocity speeds becomes larger. Therefore, apparently main circulation area is generated between the hub and the blade tip.
\end{abstract}

\section{Introduction}

The mixer is widely used in architecture, chemical industry, food and other industries. With the development of CFD, numerical simulation method is used to study the flow characteristic in mixing [1-3], which not only saves the research funding but also gets the date that can't be gotten from the experiment. Multiphase flow structures under different conditions are calculated by the large eddy simulation and multiphase flow model. At the same time the inner flow characteristic is researched combined with hydrodynamic mechanism, which provides the theoretic foundation for the mixing preparation.

\section{Turbulence model}

Large eddy simulation. In LES flow features which are larger than the grid size are solved for explicitly, that is they are not modelled, whereas flow structures which are smaller than the grid size are modelled, using SGS models. The mathematical model employed here is based on the solution of conservation equations for mass, momentum and a conserved scalar. The Favre averaged equations for mass and momentum are as follows:

$$
\begin{aligned}
& \frac{\partial \bar{\rho}}{\partial t}+\frac{\partial}{\partial x_{j}}\left(\bar{\rho} \tilde{u}_{j}\right)=0 \\
& \frac{\partial \bar{\rho} \tilde{u}_{i}}{\partial t}+\frac{\partial}{\partial x_{j}}\left(\bar{\rho} \tilde{u}_{j} \tilde{u}_{i}\right)=-\frac{\partial \bar{p}}{\partial x_{i}}+\frac{\partial}{\partial x_{j}}\left(\mu_{e f f}\left(\frac{\partial \tilde{u}_{i}}{\partial x_{j}}+\frac{\partial \tilde{u}_{j}}{\partial x_{i}}\right)\right)+\bar{\rho} g_{i}
\end{aligned}
$$

$\mu_{\mathrm{t}}$ is calculated using the dynamic model of Lilly which is similar to the Smagorinsky SGS model, i.e. Cs is not a constant, but is computed dynamically.

Mixture model. The mixing process between water and low density particles are described by mixture model, which can simulate the multi-phase flow with different velocities. Assuming local equilibrium in the spatial scale, continuity equation of the mixture model is as follows: 


$$
\frac{\partial}{\partial t}\left(\rho_{m}\right)+\nabla \cdot\left(\rho_{m} \vec{v}_{m}\right)=0
$$

The momentum equation of the mixture can be obtained by the sum of momentum equation of all phases, which can be described as follows:

$$
\frac{\partial}{\partial t}\left(\rho_{m} \vec{v}_{m}\right)+\nabla \cdot\left(\rho_{m} \vec{v}_{m} \vec{v}_{m}\right)=-\nabla p+\nabla \cdot\left[\mu_{m}\left(\nabla \vec{v}_{m}+\nabla \vec{v}_{m}^{T}\right)\right]+\rho_{m} \vec{g}_{m}+\vec{F}+\nabla \cdot\left(\sum_{k=1}^{n} a_{k} \rho_{k} \vec{v}_{d r, k} \vec{v}_{d r, k}\right)
$$

$\rho_{m}$ is the mixture density, $\vec{v}_{m}$ is the average velocity of the mass, $\mu_{m}$ is the mixed viscosity coefficient, $\vec{F}$ is the body force, $n$ is number of phases, $a_{k}$ is $k$ phase volume fraction, $\rho_{k}$ is $k$ phase density and $\vec{v}_{d r, k}$ is $k$ phase flowing speed.

The slip velocity $\vec{v}_{q p}$ is defined as the velocity of the second phase $p$ relative to principal phase $q$. And $\vec{v}_{q p}$ is as follows:

$\vec{v}_{q p}=\vec{v}_{p}-\vec{v}_{q}$

The relation of flowing speed and slip velocity is as follows:

$$
\vec{v}_{d r, k}=\vec{v}_{q p}-\sum_{k=1}^{n} \frac{a_{k} \rho_{k}}{\rho_{m}} \vec{v}_{q k}
$$

The volume fraction equation of the second phase can be got from the continuity equation.

$$
\frac{\partial}{\partial t}\left(a_{p} \rho_{p}\right)+\nabla \cdot\left(a_{p} \rho_{p} v_{m}\right)=-\nabla \cdot\left(a_{p} \rho_{p} v_{d r, p}\right)
$$

\section{Computational model and boundary condition}

The standard stirred tank is taken as the subject in the study. The diameter $\mathrm{T}$ is $0.5 \mathrm{~m}$, and the liquid depth $\mathrm{H}$ equals to $\mathrm{T}$. With the six-blade ruston turbine, diameter of the paddle is $\mathrm{D}$, width $\mathrm{W}$ is $\mathrm{D} / 5$, the length $L$ is $D / 4$ and the thickness $d$ is $2 \mathrm{~mm}$. The structure is shown in Fig. 1 . The flow is discrete due to unstructured meshes. Grid treatments are used for paddle, interface and the area near the wall to improve calculation accuracy. The number of grid nodes is 528546. Schematic diagram of grid is shown in Fig.1.

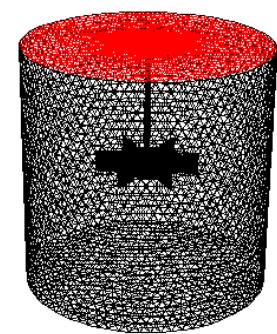

Fig 1 Diagram of computational model and meshes

The equation is discrete by the finite volume method and SIMPLE algorithm is used for the pressure-velocity coupling. At the same time mixture model is used to simulate the mixture separation. The cylinder wall and the paddle are set as Wall and moving of the cylinder wall is set as Moving Wall. The rotational axis starts from the origin in different conditions around the axis. Moving Reference Frame is used to simulate fluid motion and the direction of rotation is set around the axis direction. In this paper water and solid liquid mixtures are the calculated mediums. For easy figures, the medium is taken as a single component liquid with the higher density and viscosity than water. Rated speeds are $30 \mathrm{r} / \mathrm{min}, 60 \mathrm{r} / \mathrm{min}$ and $120 \mathrm{r} / \mathrm{min}$.

\section{Computing result and analysis}

To study the distribution regularities and flow characteristic of the two fluids in the mixing, unsteady numerical simulation of the two phase fluids in the stirred tank is taken. Distributions of the surface of fluids at different time with different speeds are shown in Fig.2 and Fig.3. With the speed 
increasing, interface of the two fluids develops from cone to cylinder. With the speed of 12rad/s, the interface starts from the paddle and upwards in a spiral to the inverted cone. As mixing time goes by, the interface rotates with the paddle. With the speed of $24 \mathrm{rad} / \mathrm{s}$, the bottom of the interface swaddles the paddle. Then the cylindric interface is formed from bottom to top, which is because with the increasing speed, the increasing centrifugal force causes the more obvious centrifugal effect of the interface.

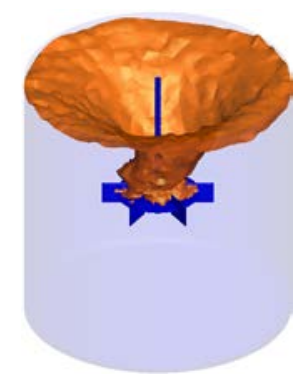

(a) $5 \mathrm{~s}$

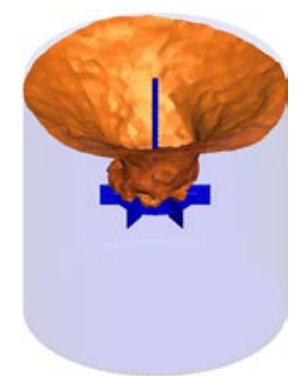

(b) $10 \mathrm{~s}$

Fig 2 Distributions of the fluid surface at different time with the speed of $12 \mathrm{rad} / \mathrm{s}$

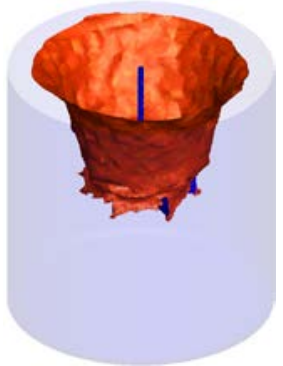

(a) $5 \mathrm{~s}$

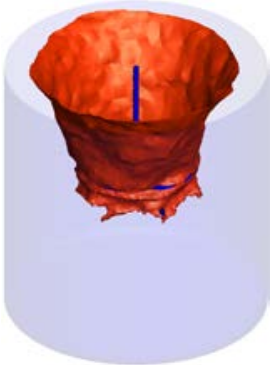

(b) $10 \mathrm{~s}$

Fig 3 Distributions of the fluid surface at different time with the speed of $24 \mathrm{rad} / \mathrm{s}$

The vorticity distributions at different time with different speeds are shown in Fig.4 and Fig.5. There are the obvious circulation areas among the paddles. Velocity near the blade tip is high. And a large number of small vortexes are formed because of the strong turbulence. It can be seen that vortexes near the blade tip increase with the speed. With the speed of $24 \mathrm{rad} / \mathrm{s}$, vortex layer is formed in the all blade disc, where the flues mix violently.

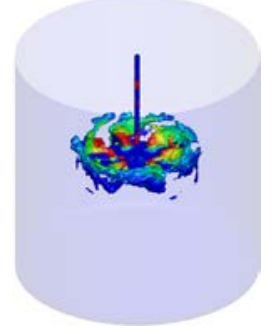

(a) $5 \mathrm{~s}$

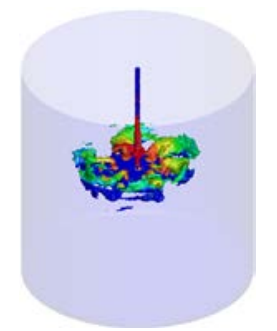

(b) $10 \mathrm{~s}$

Fig 4 The vorticity distributions at different time with $12 \mathrm{rad} / \mathrm{s}$

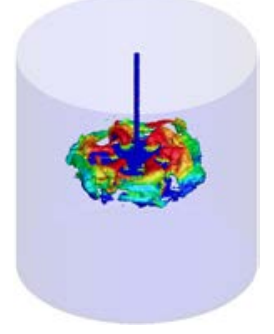

(a) $5 \mathrm{~s}$

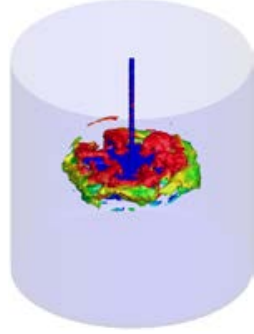

(b) $10 \mathrm{~s}$

Fig 5 The vorticity distributions at different time with $24 \mathrm{rad} / \mathrm{s}$

Pressure distributions of the flow field under the different work conditions with different speeds are analyzed in Fig.6. Pressure difference decreases gradually with the increasing position, which shows that mixing effect is weak in the area far away from the paddle. Pressure difference increases gradually with the increasing speed, which shows the suitable increasement of speed can improve the 
mixing effect. The radial velocity distributions with the two speeds are showed in Fig.7. There are contrary velocity peeks between the hub and the blade tip. And with the speed increasing, difference between the radial velocity speeds becomes larger. Therefore, apparently main circulation area is generated between the hub and the blade tip.

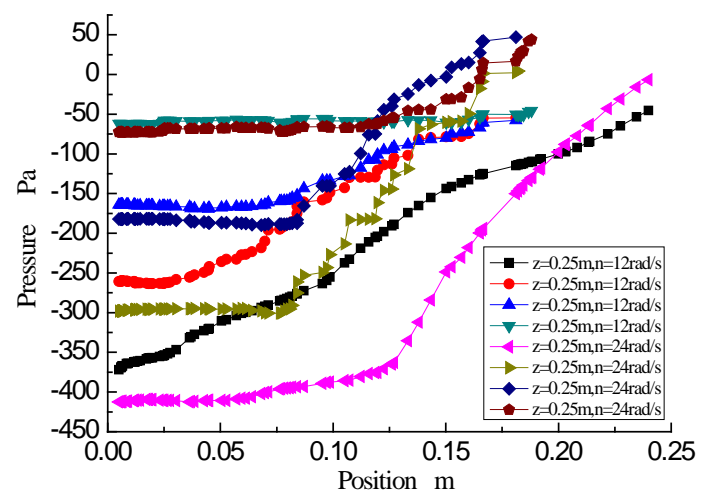

Fig 6 The radial pressure distributions with different speeds

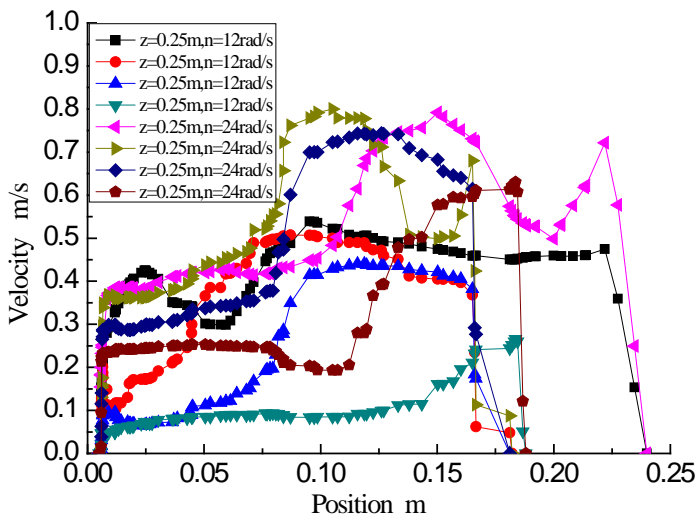

Fig 7 The radial velocity distributions with different speeds

\section{Conclusions}

Unsteady flow and the interface distribution in the stirred tank are studied by the large eddy simulation and multiphase flow model. The result shows that the two-phase interface starts from the paddle. Finally inverted cone interface is generated by the screw move. With mixing time passing by, the interface rotates with the paddle. Centrifugal effect is more obvious along with the speed rise. There is an apparently circulation area among the blades. Velocity near the blade tip is high. And a large number of small vortexes are formed because of the strong turbulence. Then fuels around are carried to other areas by the vortex motion. There are contrary velocity peeks between the hub and the blade tip. And with the speed increasing, difference between the radial velocity speeds becomes larger. Therefore, apparently main circulation area is generated between the hub and the blade tip.

\section{Acknowledgements}

This work was financially supported by the scientific research foundation of Shandong province Outstanding Young Scientist Award (No: BS2013NJ017) and Qingdao science and technology program of basic research projects (No: 13-1-4-210-jch and 13-1-4-248-jch)

\section{References}

[1] Hristov H V, Boden S, Hampel U, et al., A study on the two-phase flow in a stirred tank reactor agitated by a gas-inducing turbine. Chemical Engineering Research and Design, Vol. 86(2008): p. 75-81.

[2] Chen Y, Jiang H, Bao Y Y, Huang X B., Turbulence properties of solid-liquid flow in the near-wall region stirred tank. Journal of Chemical Engineering of Japan, Vol. 44(2011): p. 224-232.

[3] Delafosse A, Line A, Morcjain J, et al., LES and URANS simulation of hydrodynamics in mixing tank: Comparison to PIV experiments. Chemical Engineering Research and Design, Vol. 86(2008): p. 1322-1330. 